\title{
REVISIÓN
}

\section{Epilepsia mioclónica progresiva tipo Lafora y los casos diagnosticados en Costa Rica}

\section{Virginia Solís}

Escuela de Biología, Universidad de Costa Rica, San José, Costa Rica. Fax (506) 207 4216; msolis@biologia.ucr.ac.cr

\author{
Recibido 21-XI-2003. Corregido 29-VIII-2004. Aceptado 31-VIII-2004.
}

\begin{abstract}
Lafora's progressive myoclonus epilepsy and diagnosed cases in Costa Rica. A review of the main clinical, pathologic and genetic aspects of progressive myoclonus epilepsy Lafora type was undertaken. The diagnosed cases of this disorder in Costa Rica are mentioned. Rev. Biol. Trop. 52(3): 571-584. Epub 2004 Dic 15.
\end{abstract}

Key words: Lafora disease, myoclonic epilepsy, Costa Rica.

Palabras clave: enfermedad de Lafora, epilepsia mioclónica, Costa Rica.

La Enfermedad de Lafora (EL) es una epilepsia mioclónica progresiva con mal pronóstico. El doctor Gonzalo Lafora se encontraba estudiando cerebros en autopsias de pacientes con epilepsia mioclónica y demencia, cuya edad de inicio había sido antes de los diecinueve años, cuando observó unas estructuras esféricas, con dos capas, a veces tan grandes como la neurona que las contenía (Lafora 1911). Estos corpúsculos sirvieron después para distinguir esta condición de los otros tipos principales de epilepsia mioclónica, en los cuales no se encuentra, a saber: Enfermedad de Unverricht-Lundborg (Unberricht 1891, Lundborg 1903), sialidosis tipo I (Federico et al. 1980), citopatías mitocondriales (Fukuhara et al. 1980) y las formas adultas de la ceroidolipofuscinosis neuronal (Kufs et al. 1925).

El paciente típico con EL es un joven normal que comienza a presentar uno o más de los síntomas que se describirán a continuación. Se pueden observar deprimidos y desempeñarse mal en la escuela, o pueden sufrir una convulsión generalizada. Las convulsiones occipitales y mioclónicas son rasgos fundamentales de la enfermedad. La mioclonía (sacudida muscular súbita) puede ser fragmentaria, simétrica o generalizada. Ocurre en el descanso y es exagerada por la agitación, la acción o por la estimulación fótica. La mioclonía usualmente desaparece con el sueño. Ocurren sucesiones de mioclonías masivas, las cuales imitan convulsiones generalizadas y durante las cuales se conserva la conciencia de manera relativa (Van Heycop Ten Ham 1974, Roger et al. 1967, Roger et al. 1992). La mioclonía es la primera razón para la dependencia temprana de una silla de ruedas anterior a cualquier déficit motor. Las convulsiones occipitales con mucha frecuencia se presentan con ceguera transitoria, alucinaciones visuales simples o complejas, convulsiones fotomioclónicas o fotoconvulsivas o migraña clásica con escotomatas centelleantes (Van Heycop Ten Ham 1974, Roger et al. 1983, Roger et al. 1992).

En la mayoría de los pacientes, la enfermedad se establece entre los 12 y los 17 años, pero muchos pueden presentar convulsiones aisladas mucho antes, durante la infancia o la niñez (Van Heycop Ten Ham 1974, Roger et al. 1992, Acharya et al. 1995, Minassian 2000b). Muy raramente la enfermedad, que empeora progresivamente, comienza alrededor de los 6 años (Roger et al. 1992, Minassian 
2000b). Una vez iniciada, tiene una tasa variable de empeoramiento clínico, el cual depende de la severidad de la epilepsia. Las convulsiones y la mioclonía responden temporalmente al tratamiento, pero gradualmente llegan a ser intratables. La frecuencia de las convulsiones generalizadas es variable, pero todos los pacientes desarrollan mioclonías casi continuas durante las horas de vigilia y convulsiones frecuentes del lóbulo occipital. Se produce demencia progresiva, agitación, sicosis, agotamiento muscular y fallo respiratorio (Van Heycop Ten Ham 1974, Delgado Escueta et al. 2001, Ganesh et al. 2002b). La disartria y ataxia aparecen temprano durante el transcurso de la enfermedad, pero la espasticidad se establece sólo en los estados más tardíos. A mitad de los años veinte la mayoría de los pacientes presentan mioclonías continuas, están en un estado vegetativo y deben ser alimentados por sonda. Algunos logran mantener relaciones mínimas con la familia, tales como reflejos parecidos a sonrisas después de que se les halaga. Los pacientes que no son alimentados por sonda tienen aspiraciones frecuentes debido a las convulsiones y es común que mueran debido a una neumonía por broncoaspiración (Minassian 2002). Se presenta un proceso no-opoptótico inusual de neurodegeneración caracterizado por una desintegración generalizada de las organelas celulares (Van Heycop Ten Ham 1974, Ganesh et al. 2002a). La muerte sobreviene alrededor de 10 años después del comienzo de la enfermedad (Van Heycop Ten Ham 1974, Delgado Escueta et al. 2001, Ganesh et al. 2002b).

Los síntomas clínicos son precedidos por anormalidades en el electroencefalograma, como se ha observado en los hermanos jóvenes de los casos confirmados (Van Heycop Ten Ham 1974, Minassian 2000b).

\section{PATOLOGÍA}

En la biopsia de cerebro, hígado, músculo y piel se detecta la presencia de los cuerpos de Lafora (CL) (Lafora 1911, Lafora y Gluck 1911, Harriman y Millar 1955, Roger et al.
1967, Van Heycop Ten Ham 1974, Nishimura et al. 1980, Roger et al. 1983, Busard et al. 1986, Busard et al. 1987, Kobayashi et al. 1990, Roger et al. 1992, Berkovic et al. 1993, Drury et al. 1993, Acharya et al. 1995, Minassian et al. 1996, Cavanagh 1999, Minassian et al. 2000b), pero son más abundantes en los órganos con un metabolismo mayor de la glucosa: cerebro, corazón, hígado y músculo esquelético (Van Heycop Ten Ham 1974), y son más prominentes en el hígado y el músculo (Harriman y Millar 1955, Nishimura et al. 1980, Carpenter y Karpati 1981, Busard et al. 1987).

Los cuerpos de Lafora se encuentran en el Sistema Nervioso Central principalmente en las neuronas (Van Heycop Ten Ham 1974, Cavanagh 1999). Su ámbito de tamaño va de 3 a 40 y a menudo ocupan todo el citoplasma. Tienen un centro denso y una periferia menos densa y se tiñen intensamente con PAS. Esa tinción también revela gránulos pequeños semejantes a polvo que están compuestos del mismo material que los cuerpos de Lafora más grandes. Estos últimos están cerca del núcleo y los gránulos parecidos a polvo dentro de las dendritas. Se ha estimado que el tamaño acumulado de los gránulos dendríticos excede al de los cuerpos de Lafora cercanos al núcleo. Los axones raramente contienen cuerpos de Lafora o acumulaciones positivas para la reacción de PAS (Cavanagh 1999). La reacción positiva con PAS indica un contenido importante de carbohidrato. Los estudios bioquímicos han mostrado que los cuerpos de Lafora son agregados de fibras compuestas de unidades repetitivas de glucosa (poliglucosanos). Estos se diferencian del glucógeno en que pierden el patrón de ramificación normal, el cual le permite al glucógeno entrar en suspensión en el citoplasma, además están densamente empacados, son insolubles y están fosforilados (Yokoi y Austin 1968, Sakai et al. 1970, Yokoi et al. 1975). Los cuerpos de Lafora también contienen hasta un $30 \%$ de proteína no caracterizada (Sakai et al. 1970). Su estudio por medio de microscopio electrónico indica que están hechos de fibras cortas de 50 a $100 \AA$ de diámetro. Muchas fibras parecen 
estar en asociación física con el retículo endoplasmático o los ribosomas (Collins et al. 1968, Toga et al. 1968).

Lo más probable es que los CL se originen por un defecto en el metabolismo del glucógeno, porque no hay otra fuente de polímeros de glucosa en los tejidos animales (Roger et al. 1967, Collins et al. 1968, Toga et al. 1968, Sakai et al. 1970, Nikaido et al. 1971, Nishimura et al. 1980, Robitaille et al. 1980, Hays et al. 1981, Baumann et al. 1983, Roger et al. 1983, Busard y Renier 1986, Busard et al. 1987, Kobayashi et al. 1990, Berkovic et al. 1993, Drury et al. 1993, Cavanagh 1999, Minassian et al. 2000b, Minassian et al. 2001).

Se ha encontrado que el glucógeno se encuentra incrementado en el cerebro y músculos de los pacientes con Enfermedad de Lafora (Yokoi et al. 1968, Gambetti et al. 1971). Un estudio bioquímico de los cuerpos de Lafora mostró que difieren muy poco en su composición química con respecto a los cuerpos amiloideos (Sakai et al. 1970). Estos últimos son más destacados en el sistema nervioso saludable de la persona vieja y en otras especies (Cavanagh 1999). Aunque se encuentran presentes en las neuronas, los cuerpos amiloideos se encuentran principalmente en los astrocitos y en las células gliales. Por otra parte, los cuerpos de Lafora se encuentran siempre presentes en las neuronas (Van Heycop Ten Ham 1974, Ganesh et al. 2002a).

En el músculo los cuerpos de Lafora están encerrados dentro de una membrana (Carpenter et al. 1974). En el hígado, la presencia de una membrana es motivo de controversia (Nishimura et al. 1980, Carpenter y Karpati 1981). En el cerebro, la mayoría de investigadores han informado que se encuentran libres en el citoplasma (Gambetti et al. 1971, Carpenter y Karpati 1974, Neville et al. 1974, Van Heycop Ten Ham 1974, Busard et al. 1987). Cajal y colaboradores (1975) afirmaron que durante las fases tempranas de su maduración, los cuerpos de Lafora están encapsulados dentro de una estructura membranosa. En la piel, los cuerpos de Lafora pueden ser encontrados en las células mioepiteliales tanto de las glándulas ecrinas como apocrinas, pero su localización predominante es en las células ductales de las ecrinas y no de las segundas (Carpenter y Karpati 1981). La axila está llena de glándulas apocrinas y por lo tanto no ofrece ventaja, respecto a las biopsias tomadas en otros sitios. De esta manera es preferible evitar tomar biopsias de esa zona. La biopsia puede ser negativa debido a un error en la toma de la muestra (Drury et al. 1993, Minassian et al. 2000b). Una biopsia muscular negativa no es infrecuente, pero una biopsia de piel negativa es rara (Carpenter y Karpati, 1981, Busard et al. 1987).

Los ratones enfermos con EL presentan, en los estados tempranos, una difundida degeneración de las células nerviosas, en ausencia de los cuerpos de Lafora. Esto sugiere que la EL es un trastorno neurodegenerativo primario y que los cuerpos de Lafora no juegan un papel primario en la epileptogénesis (Ganesh et al. 2002a). En este sentido es importante señalar que los pacientes con enfermedad adulta de los cuerpos de poliglucosano, tienen muchas de estas inclusiones, pero no presentan epilepsia ni mioclonías (Robitaille et al. 1980). En su lugar desarrollan de forma progresiva déficits motores y sensoriales junto con demencia, un subgrupo de rasgos que son vistos en los pacientes con EL y que están también asociados con el proceso de envejecimiento (Cavanagh 1999). Lo anterior lleva a suponer que la acumulación de los poliglucosanos en las neuronas es lo que llevaría al desarrollo de estos síntomas (Ganesh et al. 2004). Se considera que el material acumulado en la epilepsia de Lafora es patogénico, debido al inicio retardado de la enfermedad y a su avance progresivo (Minassian 2002).

\section{GENÉTICA}

La Enfermedad de Lafora es de herencia autosómica recesiva y con pocas excepciones los pacientes siguen un curso clínico homogéneo, a pesar de la heterogeneidad genética de la enfermedad (Minassian et al. 1999). Se han informado unos poquísimos casos de pacientes 
que han presentado una evolución progresiva más rápida (Van Heycop Ten Han 1974, Minassian 2001, Al Otaibi et al. 2003).

El primer gen identificado como responsable de la Enfermedad de Lafora fue llamado EPM2A y fue mapeado en el cromosoma 6q24 (Serratosa et al. 1995, Sainz et al. 1997), se identificó utilizando un ensayo estándar de clonación posicional (Minassian et al. 1998, Serratosa et al. 1999) y toda su región codificante fue caracterizada (Ganesh et al. 2000). Está compuesto por cuatro exones y da lugar a una proteína de 331 aminoácidos llamada Laforina. El análisis de la secuencia de la Laforina mostró que la mitad que posee el extremo $\mathrm{N}$ terminal contiene una región de unión con carbohidrato (Minassian et al. 2000b, Ganesh et al. 2001) que se une al glucógeno in vitro (Ganesh et al. 2002b). Esta y otras regiones de unión con carbohidratos son secuencias conservadas evolutivamente que se encuentran en una variedad de proteínas desde los procariotas hasta los humanos y digieren o bien interactúan con varios carbohidratos, entre los que se incluye al almidón y al glucógeno. La Laforina no muestra homología con ninguna otra proteína conocida y además contiene un motivo de consenso para fosfatasa tirosínica proteica en su extremo carboxílico. Los estudios funcionales revelan que la Laforina es una fosfatasa activa con especificidad doble. (Ganesh et al. 2000). Ambos dominios se encuentran también presentes en los ortólogos del ratón y la rata, lo cual sugiere su conservación funcional en los mamíferos (Ganesh et al. 2001).

La localización subcelular de la Laforina fue establecida en cultivo de tejidos no neuronales. Se la encontró situada en el Retículo Endoplasmático (Ganesh et al. 2000, Minassian et al. 2001) y en la membrana plasmática (Minassian et al. 2001). Probablemente no es una (proteína de membrana integral, porque pierde algunos de los supuestos dominios transmembrana (Minassian et al. 2000b), ni tampoco es una proteína residente dentro del Retículo Endoplasmático, porque carece de un péptido señal para el mismo (Minassian et al. 2000b). Se encontró que en el Retículo Endoplasmático la
Laforina está situada en los ribosomas en una interacción proteína-proteína con componentes ribosómicos no identificados (Ganesh et al. 2000).

El gen EPM2A codifica dos isoformas de la proteína que surgen por medio de un procesamiento alternativo del ARN y comparten un segmento común extenso, pero difieren en sus extremos C terminales (Minassian et al. 1998). El segmento común consiste de una región para la unión con los carbohidratos (Minassian et al. 2000b) y un dominio con doble especificidad fosfatásica (Ganesh et al. 2000, Minassian et al. 2000a, 2001). La isoforma A se localiza en el retículo endoplasmático rugoso (Ganesh et al. 2000, Minassian et al. 2001) y se une al glucógeno (Wang et al. 2002). La isoforma B se localiza en el núcleo (Ganesh et al. 2002c) donde se une al glucógeno y en ambos casos sus substratos fosfatásicos son desconocidos. Hasta el momento todas las mutaciones que causan Enfermedad de Lafora han sido localizadas en el segmento común a ambas isoformas. Ianzano et al. (2004) encontraron que la patobiología de la enfermedad se debe a un trastorno funcional de la isoforma A. Ellos identificaron seis nuevas mutaciones, una de las cuales c.950insT, es la primera mutación específica de la isoforma citoplasmática de la Laforina, lo cual la implica como una de las causas de la enfermedad. Además, aunque conservó su localización en el retículo endoplasmático, poseía una drástica reducción en su actividad fosfatásica. Los datos aportados por estudios recientes muestran que la alteración de la actividad fosfatásica de la Laforina es fundamental para la patogénesis de la enfermedad (Ianzano et al. 2004).

La Laforina pertenece a un grupo de más de 500 miembros de proteínas reguladoras que ajustan las funciones de sus substratos regulando el estado de fosforilación de algunos residuos claves de tirosina (Denu et al. 1996, Tonks y Neel 1996, Hafen 1998, Martell et al. 1998). Todas estas enzimas contienen el motivo de la secuencia de aminoácidos HCxxGxxRS/T, usualmente en la mitad carboxi terminal (x representa cualquier aminoácido) 
(Denu et al. 1996). Este motivo y las secuencias circunvecinas forman el dominio catalítico utilizado en la reacción fosfatásica (Tonks y Neel 1996). Aunque todas ellas utilizan el mismo mecanismo catalítico, cada una tiene sólo unos pocos sustratos blanco. Esta especificidad es usualmente conferida por la mitad del extremo amino, cuya secuencia es única para una enzima en particular (Hafen 1998). Estas proteínas con doble especificidad pueden ejercer su función reguladora desfosforilando no sólo a la tirosina sino también a la serina y a la treonina (Martell et al. 1998).

Se conoce poco acerca del papel de la Laforina en el metabolismo del glucógeno, sobre la integridad neuronal y la epilepsia, así como acerca de sus proteínas asociadas o sustratos (Ianzano et al. 2003)

Con respecto al papel de la Laforina en la patogénesis de la EF se plantea la posibilidad de su participación en la regulación de la excitabilidad neuronal mediada por la insulina (Abbott et al. 1999, Zhao et al. 1999, Man et al. 2000). En el cerebro, la insulina está además involucrada en la regulación de la transmisión sináptica. El receptor de la insulina está localizado en las dendritas y en otros sitios terminales postsinápticos en el cuerpo de la neurona. Una quinasa de la tirosina, es la que regula los receptores AMPA y GABA-A en la sinapsis, cuando es activada por la insulina. (Abbott et al. 1999, Zhao et al. 1999, Man et al. 2000). No se conoce, hasta qué punto esta acción de la insulina diverge hacia sus varias vías. Es posible que la Laforina actúe sobre un punto común, influyendo ambas, por lo tanto, sobre el metabolismo del glucógeno, lo cual resultaría en la producción de los Cuerpos de Lafora y la transmisión sináptica mediada por la insulina daría lugar a la epilepsia (Minassian 2002).

La ramificación del glucógeno requiere la acción coordinada de varias enzimas, cuyos defectos pueden ser de importancia para la EL. Existen cuatro enzimas citoplasmáticas que actúan directamente sobre la partícula de glucógeno. La glucógeno sintasa añade unidades de glucosa-6-fosfato para alargar un hilo de glucógeno. La enzima ramificante del glucógeno detecta el alargamiento y transfiere seis o más residuos de glucosa del extremo de la cadena larga a otros segmentos de la partícula, de este modo mantiene su redondez y previene la elongación asimétrica de las ramas. Con respecto a su catabolismo, cuando se requiere glucosa la glucógeno fosforilasa remueve una unidad a la vez y se detiene y separa cuando quedan cuatro unidades. La enzima desramificante remueve este tetrasacárido y lo coloca en una cadena más larga, para su posterior digestión por la glucógeno fosforilasa (Chen y Burchell 1995).

Debido al orden en que actúan las enzimas catabólicas, no se esperaría que sus deficiencias o hiperactividades resultaran en cadenas de glucógeno asimétricas largas (poliglucosanos). Al contrario, las enzimas anabólicas necesitan actuar de una manera equilibrada determinada. El alargamiento desbalanceado de la cadena debido a una ramificación inadecuada, resultaría en la producción de poliglucosanos, como fue propuesto por DiMauro et al. en 1971. Raben et al. (2000), proporcionaron respaldo a la teoría de DiMauro et al. (1971). Ellos realizaron un experimento en el cual utilizaron un ratón "knockout" que expresaba de manera tardía la enfermedad de Pompe. El cruce de ese ratón con un ratón transgénico que sobreexpresaba la glucógeno sintasa en el músculo esquelético, produjo descendientes que desarrollaron Enfermedad de Pompe a una edad más temprana, pero que además acumularon cuerpos típicos de poliglucosano en el músculo. De esta manera se mostró que un nivel aumentado de glucógeno sintasa, en presencia de un nivel normal de la enzima ramificante del glucógeno, conduce a una acumulación de poliglucosano.

La glucosa-6-fosfato es el activador alostérico más potente de la glucógeno sintasa (Villar-Palasi 1997). Además su actividad está estrechamente regulada a través de fosforilación en varios residuos específicos de serina. Este estado de fosforilación puede ser afectado por varias quinasas y fosfatasas, entre las cuales están la quinasa de la caseína I y II , la quinasa dependiente del cAMP, la glucógeno 
sintasa quinasa y la protein-fosfatasa-1 (Roach et al. 1991). Estas enzimas son a su vez reguladas por fosforilación (Roach et al. 1991, Woodgett 1994) y son probablemente elementos corriente abajo de vías de señales de transducción iniciadas por la insulina, glucagón, hormonas adrenérgicas y otros factores. La Laforina por ser una fosfatasa tirosínica proteica podría estar involucrada en la fosforegulación de las anteriores u otras enzimas que controlan a la glucógeno sintasa. No se espera que la Laforina actúe directamente sobre la glucógeno sintasa porque la desfosforilación inhibe a esta última (Roach et al. 1991).

Las quinasas y fosfatasas que controlan la actividad de la glucógeno sintasa son enzimas multipropósito empleadas por muchas cascadas celulares de transducción de señales. A través de la unión con proteínas específicas, que las llevan al sitio en el cual son requeridas, adquieren especificidad para realizar funciones particulares, como por ejemplo el control de la actividad de la glucógeno sintasa. Ejemplos de esto lo constituyen varias proteínas de unión con protein-fosfatasas-1 que contienen regiones para la unión con carbohidratos que dirigen a la protein-fosfatasa-1 a la glucógeno sintasa sobre la partícula de glucógeno (DiMauro et al. 1971). Es importante señalar que la Laforina también contiene una región para la unión con carbohidratos, similar a los correspondientes de las fosfatasas proteicas.

La partícula de glucógeno y su glucógeno sintasa estrechamente unida a él (DiMauro et al. 1971, Chamlian et al. 1989) la glucógeno sintasa quinasa (Angenstein et al. 1998), la protein-fosfatasa-1 (Liu y Brautigan 2000) y la Laforina, se asocian con el Retículo Endoplasmático. Por lo tanto, se dan en ese lugar las condiciones físicas para que ocurran interacciones que podrían resultar en una hiperfunción de la glucógeno sintasa asociada al Retículo Endoplasmático y las deposiciones de poliglucosanos asociados a éste que se observan en la EL (Minassian 2002).

$\mathrm{Al}$ igual que sucede en los humanos, los mutantes nulos para el gen Epm2a en los ratones, desarrollan cuerpos de Lafora, neurodegeneración, ataxia, epilepsia mioclónica y respuesta deteriorada en el comportamiento (Ganesh et al. 2002a). Los cuerpos de Lafora en ratones afectados aumentan de tamaño y número con la edad. Las regiones más afectadas incluyen el hipocampo, cerebelo, corteza cerebral, tálamo y el tallo cerebral, lo cual se correlaciona con los sitios de expresión de la Laforina (Ganesh et al. 2001, Ganesh et al. 2002a). Debido a que los cuerpos de poliglucosano son producidos normalmente en las células nerviosas (Cavanagh 1999) y a que la Laforina se une al glucógeno in vitro (Ganesh et al. 2002b) se postuló que la Laforina podría intervenir en la disposición de los cuerpos de Lafora por unión directa a ellos (Ganesh et al. 2002a). En el estudio publicado por Ganesh et al. (2004) se demostró que esto realmente ocurre. Ellos también ensayaron in vitro los efectos de cinco de las mutaciones con sentido equivocado, sobre la afinidad de la Laforina por los cuerpos de Lafora. De ellas sólo una de las mutaciones (W32G) afectó la unión con cuerpos de Lafora purificados. Con base en esas observaciones propusieron la existencia de una vía metabólica del glucógeno mediada por la Laforina, que regula la distribución de las inclusiones patogénicas de poliglucosanos (Ganesh et al. 2004). La proteína mutante W32G, mostró menor afinidad tanto para el glucógeno como para los cuerpos de Lafora, lo cual implica que el mecanismo de unión a ambos es idéntico y que la mutación afectó la conformación proteica, produciendo una pérdida de su función (Ganesh et al. 2004).

Hasta el año 2000, se habían descrito más de 36 mutaciones distintas en la región codificante del gen EPM2A (Minassian et al. 1998, Serratosa et al. 1999, Gómez-Garre et al. 2000, Minassian et al. 2000a, Minassian et al. 2000b, Ganesh et al. 2002b). 13 de los 14 aminoácidos afectados por mutaciones inactivantes con sentido equivocado, están conservados en todas las laforinas animales descritas. La región más sobresaliente, en este sentido, es el área del dominio que se une a los carbohidratos (aminoácidos 1-119). Ganesh et al. (2004) encontraron que al menos cuatro mutaciones 
con sentido equivocado, en el dominio de unión a los carbohidratos de la Laforina, no influyen sobre su afinidad por el glucógeno y los cuerpos de Lafora. Esto podría significar que ese dominio podría también estar involucrado en otros procesos celulares y que las mutaciones sin sentido en el mismo, podrían afectar algunas de esas funciones. En un estudio anterior se mostró que el mutante R108C de ese dominio no se pudo unir con los poliribosomas (Ganesh et al. 2002b), demostrando que esa región tiene múltiples funciones.

Las mutaciones localizadas en el exón 1 del gen EPM2A parecen estar asociadas con un subfenotipo que consiste en un déficit cognitivo de inicio temprano (Ganesh et al. 2002b).

Según Ganesh et al. (2004), su hallazgo de que la Laforina se une con los cuerpos de Lafora hace surgir modelos interesantes acerca de la patogénesis de la enfermedad. Los poliglucosanos neuronales parecen ser producidos normalmente en el soma y migran hacia los axones donde se acumulan, agrandan con la edad y llegan a ser detectables microscópicamente (Cavanagh 1999). Su destino final es desconocido, pero existen evidencias que sugieren que las inclusiones son transferidas a la neuroglia (Cavanagh 1999, Powel et al. 1979). Se postula su pasaje al fluido cerebroespinal como la última ruta de desecho del poliglucosano (Cavanagh 1999). La Laforina por su afinidad al glucógeno, podría unirse a las inclusiones de poliglucosano y facilitar su transporte fuera de la neurona. Otra alternativa es que podría facilitar la degradación de las inclusiones de poliglucosano, reclutando factores que están involucrados en el metabolismo del glucógeno, por ejemplo la glucógeno sintasa (Wang et al. 2002). Si no hay Laforina o ha perdido su función, las inclusiones de poliglucosano podrían detenerse en las neuronas y agrandarse para formar cuerpos de Lafora en una edad temprana (Ganesh et al. 2004). Como ya se mencionó, las propiedades bioquímicas de los cuerpos de Lafora son casi las mismas de los cuerpos amiloideos que se forman durante el envejecimiento. Esto sugiere que probablemente ambos se originan por el mismo mecanismo (Sakai et al. 1970). Ambas inclusiones son ubiquitinadas y positivas para productos finales con glicación avanzada. Eso sugiere que estas moléculas de azúcar polimerizadas forman la base para atrapar y secuestrar productos de daño oxidativo y productos no degradables (Iwaki et al. 1996, Schmith et al. 2000, Ganesh et al. 2002).

Recientemente se publicó el hallazgo de otro gen asociado con la EL llamado NHLRC1 (EPM2B), en el cromosoma 6p22.3 y que posee un sólo exón (Chan et al. 2003a). Este gen tiene todos los rasgos propuestos de la secuencia de consenso de un sitio de inicio de la traducción eucariótico en su extremo 5' y dos señales de poliadenilación en su extremo 3'. El análisis con Northern-Blot indicó la presencia de NHLRC1 en la forma de dos transcritos de $1.5 \mathrm{~kb}$ y $2.4 \mathrm{~kb}$, en todos los tejidos analizados, que incluyó subregiones específicas del cerebro. NHLRC1 codifica una proteína de 395 aminoácidos, llamada Malina. Esta tiene dedos de zinc del tipo RING y seis dominios repetidos NHL de interacción proteína-proteína. El motivo dedos de zinc RING (C-X2-C-X16-C$\mathrm{X} 1-\mathrm{H}-\mathrm{X} 2-\mathrm{C}-\mathrm{X} 2-\mathrm{C}-\mathrm{X} 13-\mathrm{C}-\mathrm{X} 2-\mathrm{C})$ entre los residuos 26 y 71, consistente con la secuencia señal (C-X2-C-X9-39-C-X1-3-H-X2-3-H-X2C-X4-48-C-X2-C) del tipo RING-HC (Freemont 2000). La presencia de dedos de zinc RING predice una función de ubiquitin-ligasa E3 (Freemont 2000, Hatakeyana y Nakayama 2003). La unión E3 es la etapa final de la vía de la ubiquitina, que transfiere ubiquitina desde E2, ya sea directamente o a través de proteínas adaptadoras, a substratos específicos para ser iniciados para su remoción por el sistema proteosómico (Hatakeyana y Nakayama 2003). Se identificaron (Chan et al. 2003b) seis motivos NHL (Slack y Ruvkun 1998) basándose en un motivo de aproximadamente 44 residuos ricos en glicina y aminoácidos hidrofóbicos depositados con un grupo de residuos cargados. Se secuenció el gen NHLRC1 en una cohorte de 34 probandos con EL que no poseían mutaciones en el gen EPM2A (Chan et al. 2003b). Se identificaron 17 diferentes alteraciones en la secuencia del ADN en 26 familias, 
que no estuvieron presentes en 100 cromosomas de control. Entre ellas se encontraban ocho deleciones y una inserción que producían cambios en el marco de lectura (frameshift), siete mutaciones con sentido equivocado y una sin sentido, en forma recesiva homocigota (18 familias) o heterocigota compuesto ( 8 familias). Las cuatro familias Canadienses-Francesas portaban cambios homocigotos $76 \mathrm{~T} \rightarrow \mathrm{A}$ que producían una sustitución de cisteína por serina, en uno de los siete residuos de serina conservados esenciales para la habilidad de unión al zinc de los dedos RING. Otra familia portaba una deleción homocigota de 2 pares de bases (1048-1049 delGA) que producía un cambio en el arreglo de lectura después del dominio quinto NHL. Al igual que la Laforina se localiza en el Retículo Endoplasmático. La Malina está probablemente involucrada por interacción a través de sus dominios NHL, seguido por la remoción mediada por la ubiquitina de blancos reguladores, y tiene el rol crucial, junto con la Laforina, de resguardar a las neuronas de los cuerpos de Lafora y de la epilepsia (Chan et al. 2003b).

En la cohorte de 102 familias con EF que ha sido estudiada hasta el momento por Chan et al. (2004b) el 48\% han presentado mutaciones en el gen EPM2A y $40 \%$ en EPM2B. El $12 \%$ restante o poseen mutaciones en regiones no codificantes de estos dos genes, que no han podido ser identificadas con los métodos utilizados, o tienen mutaciones en otro(s) gen(es) (Chan et al. 2004b). La utilización del análisis de haplotipos y ligamiento genético, en los miembros de una familia con varios afectados, les permitió mostrar la posible existencia de un tercer locus.

La presencia de los cuerpos de Lafora en esta enfermedad, indica la existencia de una vía bioquímica, aún desconocida, relacionada con el metabolismo del glucógeno, cuyos bloqueos resultan en la producción de acumulaciones celulares parecidas al almidón. Hasta ahora se han identificado cinco componentes de esta vía: la Laforina (Minassian et al. 1998), la Malina (Chan et al. 2003b), y tres proteínas que interactúan con la Laforina: EPM2AIP1
(Ianzano et al. 2003), HIRIP5 (Ganesh et al. 2003) y R5 (Fernández-Sánchez et al. 2003).

El gen EPM2AIP1 posee un gran exón de 1824 nucleótidos y tiene regiones alternativas 3 ' no traducidas. Fue mapeado en el cromosoma 3p22.1. La proteína que codifica es de función desconocida. Los estudios realizados mostraron que se encuentra localizada junto con la Laforina en el mismo compartimiento celular en el cual se depositan los poliglucosanos de la EF. La Laforina se une al glucógeno por medio de una región de unión con los carbohidratos de su extremo carboxil (Wang et al. 2002, Minassian et al. 2000b). La proteína del gen EPM2AIP1 no contiene un motivo conocido de unión para los carbohidratos y probablemente no interactúa directamente con el glucógeno. No obstante, contiene varios sitios de fosforilación y puede por lo tanto interactuar con la propiedad fosfatásica de la Laforina. En dicho estudio se encontró que las variantes deletadas de la Laforina, que perdían los dominios de unión con los carbohidratos o fosfatasas, suprimían la unión con EPM2AIP1. Esto indica que se requiere una Laforina intacta para que se produzca la interacción. El análisis del nuevo gen EPM2AIP1 se realizó en pacientes con EF que no poseían mutaciones en el gen EPM2A, ni en el primero, lo cual demostró que este nuevo gen no fue el causante de la enfermedad en estos pacientes (Ganesh $e t$ al. 2003, Ianzano et al. 2003 ).

La Laforina interactúa con la HIRIP5, que es una proteína conservada filogenéticamente poseedora de un dominio tipo NifU. Los ensayos in vitro e in vivo mostraron que la interacción es específica y que la Laforina usa probablemente su dominio N-terminal CBD-4 para interaccionar con el dominio C-terminal tipo NifU de la proteína HIRIP5. Ésta última es una proteína citosólica y es expresada ubicuamente, lo que podría reflejar una función homeostática. La presencia de un dominio tipo NifU en esta proteína hace surgir la posibilidad de que pueda estar involucrada en la homeostasis del hierro. Aunque el significado de la interacción entre HIRIP5 y la Laforina todavía no se conoce totalmente, debido a que la 
Laforina desfosforiló a la proteína HIRIP5 in vitro, la proteína HIRIP5 se visualiza como una compañera de unión a la Laforina y podría contribuir a entender la patología de la Enfermedad de Lafora (Ganesh et al. 2003).

La proteína R5 está bien estudiada y es esencial para el metabolismo del glucógeno. Se encarga de transportar y estabilizar a la glucógeno sintasa sobre la partícula del glucógeno (Fong et al. 2000). Esto resulta en la actividad coordinada con la enzima de ramificación del glucógeno. La sintasa elonga los hilos de glucógeno y la enzima de ramificación los mueve a sitios de ramificación apropiados, para mantener el crecimiento esférico del glucógeno y evitar la formación, por medio de la sintasa, de cadenas largas con una ramificación irregular (poliglucosanos) (Minassian 2002). La Laforina se une a los poliglucosanos y a éstos y al almidón preferentemente que al glucógeno (Chan et al. 2004a). Esto indica que una de las funciones de la Laforina probablemente comienza después de la aparición de los poliglucosanos (Chan et al. 2004a). Se ha propuesto que la Laforina está involucrada en el control de la calidad de la síntesis del glucógeno. Ella reconoce las estructuras de poliglucosano que aparecen durante la síntesis del glucógeno e inicia por medio de su dominio fosfatásico, mecanismos para oponerse a su continua formación o promueve su eliminación. Podría también interferir físicamente con la actividad de la glucógeno sintasa (Chan et al. 2004a). Se ha mostrado que su unión con R5 le ocupa las regiones de unión a ella y la de unión al glucógeno (Fernández 2003), y por lo tanto dispersaría el complejo R5-glucógeno sintasa necesario para una extensión adicional del hilo de glucógeno. Los cuerpos de Lafora se forman en muchos tejidos. En las neuronas se acumulan alrededor del núcleo y en las dendritas, pero no se presentan en los axones (Cavanagh 1999). La acumulación de cuerpos de Lafora en número suficiente de dendritas alrededor de los diez años, es probablemente una causa importante del inicio y progreso de la epilepsia (Minassian 2002). Surge la pregunta de qué media la acumulación dendrítica de la
Laforina y de si hay alguna función del metabolismo del glucógeno que segrega de una manera similar. La glucosa-6-fosfatasa es muy rica en el Retículo Endoplasmático dendrítico (Broadwell y Cataldo 1983) y está ausente en el axonal (Broadwell y Cataldo 1984). Eso desvía la glucosa-6-fosfato hacia el Retículo Endoplasmático, lejos de la glucógeno sintasa. La glucosa-6-fosfato es tanto el sustrato como el activador alostérico de la glucógeno sintasa, y los descensos en su concentración tienen el efecto más poderoso sobre la reducción de la actividad de la glucógeno sintasa (Villar-Palasi y Guinovart 1997). Otro rasgo de las dendritas que podría subyacer sobre el envío de la Laforina a esos sitios, es que las dendritas poseen ribosomas y los axones no (Steward y Banker 1992). En experimentos realizados con cultivo de tejidos, la Malina también se localizó en el Retículo Endoplasmático (Chan et al. 2004a). Los cuerpos de Lafora están ubiquitinados (Ganesh et al. 2002a). Es posible que la Malina realiza ese proceso bajo la influencia de la Laforina. Otra alternativa es que la Malina puede ubiquitinar enzimas de la síntesis del glucógeno (por ejemplo la sintasa) y dirigirlas hacia la degradación mediada por ubiquitina (Chan et al. 2004a). La malina a través de sus dominios NHL, seguido por una remoción de blancos reguladores mediada por ubiquitina, tiene junto con la Laforina, el papel fundamental de resguardar a las neuronas contra los cuerpos de Lafora y la epilepsia (Chan et al. 2003b). Por lo tanto, se considera que la Enfermedad de Lafora surge por una remoción inadecuada de los poliglucosanos y su subsiguiente acumulación en las dendritas, produciendo un trastorno de la función sináptica neuronal (Chan et al. 2003b).

En Costa Rica se han descrito cuatro casos, pertenecientes a dos matrimonios consanguíneos, residentes en Zarcero, provincia de Alajuela. Estas dos familias además tenían ancestros comunes (Solís 2000), lo que implica el papel de la consanguinidad en la aparición de la enfermedad. Unos años después de la muerte de esos afectados se presentó un nuevo caso, con una evolución más rápida y severa, en un 
matrimonio entre otros dos habitantes del mismo pueblo, sin parentesco aparente con las dos familias anteriores. El mismo fue diagnosticado por un médico patólogo, mediante el hallazgo de los cuerpos de Lafora (M. Vargas del Hospital San Juan de Dios, com. pers.). Próximamente será publicado con detalle. El grupo de investigación dirigido por el Dr. Berghe Minassian de Canadá, está realizando la caracterización molecular de la(s) mutación(es) presente(s) en Costa Rica.

\section{RESUMEN}

Se presenta una revisión bibliográfica de los principales aspectos clínicos, patológicos y genéticos de la epilepsia mioclónica progresiva tipo Lafora. Se hace mención a los casos de esta enfermedad diagnosticados en Costa Rica.

\section{REFERENCIAS}

Acharya, J.N., P. Satishchandra \& S.K. Shankar. 1995. Familial progressive myoclonus epilepsy: clinical and electrophysiologic observations. Epilepsia 36: 429434.

Abbott, M.A., D.G. Wells \& J.R. Fallon. 1999. The insulin receptor tyrosine kinase substrate p58/53 and the insulin receptor are components of CNS synapses. J. Neurosci. 19: 7300-7308.

Al Otaibi, S.F., B.A. Minassian, C.A. Ackerley, W.J. Logan \& S. Weiss. 2003. Unusual presentation of Lafora's disease. J. Child. Neurol. 18: 499-501.

Angenstein, F., W.T. Greenough \& I.J. Weiler. 1998. Metabotropic glutamate receptor-initiated translocation of protein kinase p90rsk to polyribosomes: a possible factor regulating synaptic protein synthesis. Proc. Natl. Acad. Sci. USA 95: 15078-15083.

Baumann, R.J., S.A. Kocoshis \& S. Wilson. 1983. Lafora disease: liver histopathology in presymptomatic children. Ann. Neurol. 4: 86-89.

Berkovic S.F., J. Cochius, E. Andermann \& F. Andermann. 1993. Progressive myoclonus epilepsies: clinical and genetic aspects. Epilepsia 34: S19-30.

Broadwell, R.D. \& A.M. Cataldo. 1983. The neuronal endoplasmic reticulum: its cytochemistry and contribution to the endomembrane system. I. Cell bodies and dendrites. J. Histochem. Cytochem. 31: 1077-1088.
Broadwell, R.D. \& A.M. Cataldo. 1984. The neuronal endoplasmic reticulum: its cytochemistry and contribution to the endomembrane system. II. Axons and terminals. J. Comp. Neurol. 230: 231-248.

Busard, B.L., W.O. Renier, F.J. Gabreels, H.H. Jasper, U.J. van Haelst \& J.L. Stooff. 1986. Lafora's disease: comparison of inclusion bodies in skin and in brain. Arch. Neurol. 43: 296-299.

Busard, H.L., W.O. Renier, F.J. Gabreels, H.H. Jasper, J.L. Slooff, A.J. Jansen \& U.J. van Haelst. 1987. Lafora disease: a quantitative morphological and biochemical study of the cerebral cortex. Clin. Neuropathol. 6: 1-6.

Busard, H.L., A.A. Gobreels-Festen, W.U. Renih, F.J. Gabreels \& A.M. Stadhouders. 1987. Axila skin biopsy: a reliable test for diagnosis of Lafora's disease. Ann. Neurol. 21: 599-601.

Carpenter, S., G. Karpati, F. Andermann, J.C. Jacob \& E. Andermann. 1974. Lafora's disease: Peroxisomal storage in skeletal muscle. Neurology 24: 531-538.

Carpenter, S. \& G. Karpati. 1981. Sweat gland duct cells in Lafora disease: diagnosis by skin biopsy. Neurology 31: 1564-1568.

Carpenter, S. \& G. Karpati. 1981. Ultrastructural findings in Lafora disease. Ann. Neurol. 10: 63-64.

Cavanagh, J.B. 1999. Corpora amylacea and the family of polyglucosan diseases. Brain Res. Rev. 29: 265-295.

Chamlian, A. L., Benkoel, D. Minko, T. Njee \& J.M. Gulian. 1989. Ultrastructural heterogeneity of glycogen in human liver. Liver 9: 346-350.

Chan, E.M., D.E. Bulman, A.D. Paterson, J. Turnbull, E. Andermann, F. Andermann, G.A. Rouleau, A.V. Delgado-Escueta, S.W. Scherer \& B.A. Minassian. 2003a. Genetic mapping of a new Lafora progressive myoclonus epilepsy locus (EPM2B) on 6p22. J. Med. Genet. 40: 671-675.

Chan, E.M., E.J. Young, L. Ianzano, I. Munteanu, X. Zhao, C.C. Christopoulos, G. Avanzini, M. Elia, C.A. Ackerley, N.J. Jovic, S. Bohlega, E. Andermann, G.A. Rouleau, A.V. Delgado-Escueta, B.A. Minassian \& S.W. Scherer. 2003b. Mutations in NHLRC1 cause progressive myoclonus epilepsy. Nat. Genet. 35: 125-127.

Chan, E.M., C.A. Ackerley, H. Lohi, L. Ianzano, M.A. Cortez, P. Shannon, S.W. Scherer \& B.A. Minassian. 2004a. Laforin preferentially binds to neurotoxic stach-like polyglucosans, which form in its absence in progressive myoclonus epilepsy. Hum. Molec. Genet. 13: 1117-1129. 
Chan, E.M., S. Omer, M. Ahmed, L.R. Bridges, C. Bennett, S.W. Scherer \& B.A. Minassian. 2004b. Progressive myoclonus epilepsy with polyglucosans (Lafora disease). Evidence for a third locus. Neurology 63: 565-567.

Chen, Y.T. \& A. Burchel. 1995. Glycogen Storage Diseases. pp. 935-965. In C.R. Scriber, A.L. Beaudet, W.S. Sly \& D. Valle (eds.). The metabolic and molecular bases of inherited disease. McGrau-Hill. New York.

Collins, G.H., R.R. Cowden \& A.H. Nevis. 1968. Myoclonus epilepsy with Lafora bodies: an ultrastructural and cytochemical study. Arch. Neurol. 86: 239-254.

Denu, J.M., J.A. Stuckey, M.A. Saper \& J.E. Dixon. 1996. Form and function in protein dephosphorilation. Cell 87: 361-364.

DiMauro, S., W. Trojaborg, P. Gambetti \& L.P. Rowland. 1971. Binding of enzymes of glycogen metabolism to glycogen in skeletal muscle. Arch. Biochem. Biophys. 144: 413-422.

Drury, I., M. Blaivas, B.Abou-Khalil \& A. Beydoun. 1993. Biopsy results in a kindred with Lafora disease. Arch. Neurol. 50: 102-105.

Federico, A., A. Cecio, G.A. Battini, J.C. Michalski, G. Strecker \& G.C. Guazzi. 1980. Macular cherry-red spot and myoclonus síndrome: juvenile form of sialidosis. J. Neurol. Sci. 48: 157-169.

Freemont, P.S. 2000. RING for destruction? Curr. Biol. 27: R84-87.

Fernández-Sánchez, M.E., O. Criado-García, K.E. Heath, B. García-Fojeda, I. Medrano-

Fernández , P. Gómez-Garre, P. Sanz , J.M. Serratosa \& S. Rodríguez De Córdoba. 2003. Laforin, the dual phosphatase responsable for Lafora disease, interacts with R5 (PTG), a regulatory subunit of protein phosphatase-1 that enhances glycogen accumulation. Hum. Mol. Genet. 12: 3161-3171.

Fong, N.M., T.C. Jensen, A.S. Shah, A.R. Parekh, Saltiel \& M.J. Brady. 2000. Identification of binding sites on protein targeting to glycogen for enzymes of glycogen metabolism. J. Biol. Chem. 275: 35034-35039.

Fukuhara, N., S. Tokiguchi, K. Shirakawa \& T. Tsubaki. 1980. Myoclonus epilepsy associated with raggedred fibers (mitochondrial abnormalities ):disease entity or a syndrome? Light and electron microscopic studies of two cases and a review of the literature. J. Neurol. Sci. 47: 117-133.

Ganesh, S., K. Amano, AV. Delgado-Escueta \& K. Yamakawa. 1999. Isolation and characterization of mouse homologue for the human epilepsy gene, EPM2A. Biochem. Biophys. Res. Commun. 257: 24-28.

Ganesh, S., K.L. Agarwala, K. Ueda, T. Akagi, K. Skoda, T. Usui, T. Hashikawa, H. Osada, A.V. Delgado-Escueta \& K. Yamakawa. 2000. Laforin, defective in the progressive myoclonus epilepsy of Lafora type, is a dual-specificity phosphatase associated with polyribosomes. Hum. Mol. Genet. 9: 2251-2261.

Ganesh, S., K.L. Agarwala, K. Amano, T. Suzuki, A.V. Delgado-Escueta \& K. Yamakawa. 2001. Regional and developmental expression of Epm2a gene and its evolutionary conservation. Biochem. Biophys. Res. Comun. 283: 1046-1053.

Ganesh, S., A.V. Delgado-Escueta, T. Sakamoto, M.R. Avila, J. Machado-Salas, Y. Hoshil, T. Akagi, H. Gomi, T. Suzuki, K. Amano, K.L. Agarwala, Y. Hasegawa, D.S. Bai, T. Ishihara, T. Hashikawa, S. Itohara, E.M. Cornford, H. Niki \& K. Yamakawa. 2002a. Targeted disruption of the Epm2a gene causes formation of Lafora inclusion bodies, neurodegeneration, ataxia, myoclonus epilepsy and impaired behavioral response in mice. Hum. Mol. Genet. 11: 1251-1262.

Ganesh, S., A.V. Delgado-Escueta, T. Suzuki, S. Francheschetti, C. Rigio, G. Avanzini, A. Rabinowicz, S. Bohlega, J. Bailey, M.E. Alonso, A. Rasmussen, A.E. Thomson, A. Ochoa, A.J. Prado, M.T. Medina \& K. Yamakawa. Genotype-phenotype correlations for EPM2A mutations in Lafora's progressive myoclonus epilepsy: exon 1 mutations associate with an early-onset cognitive deficit subphenotype. 2002 b. Hum. Mol. Genet. 11: 1263-1271.

Ganesh, S., T. Suzuki \& K. Yamakawa. 2002c. Alternative splicing modulates subcellular localization of laforin. Biochem. Biophys. Res. Commun. 291: 1134-1137.

Ganesh, S., N. Tsurutani, T. Suzuki, K. Ueda, K.L. Argawala, H. Osada, A.V. Delgado-Escueta \& K. Yamakawa. 2003. The Lafora disease gene product laforin interacts with HIRIP5 phylogenetically conserved protein containing NifU-like domain. Hum. Mol. Genet. 12: 2359-2368.

Ganesh, S., N. Tsurutani, T. Suzuki, Y. Hoshii, T. Ishihara, A.V. Delgado-Escueta \& K. Yamakawa. 2004. The carbohydrate binding domain of Lafora disease protein targets Lafora polyglucosan bodies. Biochem. Biophys. Res. Commun. 313: 1101-1109.

Gómez-Garre, P., Y. De Sanz, \& J.M. Serratosa. 2000. Mutational spectrum of the EPM2A gene in progressive myoclonus epilepsy of Lafora: high degree of allelic heterogeneity and prevalence of deletions. Eur. J. Hum.Genet. 8: 946-54. 
Hafen, E. 1998. Kinases and phosphatases: a marriage is consummated. Science 280: 1212-1213.

Harriman, D.G. \& J.H. Millar.1955. Progressive familial myoclonic epilepsy in three families: its clinical features and pathological basis. Brain 78: 325-349.

Hatakeyama, S. \& K.I. Nakayama. 2003. U-box proteins as a new family of ubiquitin ligases. Biochem. Biophys. Res. Commun. 302: 635-645.

Hays, A.P., M. Hallet, J. Delfs, J. Morris, A. Sotrel, M.M. Shevchuk \& S. DiMauro. 1981. Muscle phosphofructokinase deficiency. Abnormal polysaccharide in a case of late-onset myopathy. Neurology 31: 1077-1086.

Ianzano, L., X.C. Zhao, B.A. Minassian \& S.W.Scherer. 2003. Identification of a novel protein interacting with laforin, the EPM2A progressive myoclonus epilepsy gene product. Genomics 81: 579-587.

Ianzano, L., E.J. Young, X.C. Zhao, E.M. Chan, M.T. Rodríguez, M.V. Torrado, S.W. Scherer \& B.A. Minassian 2004. Loss of function of the cytoplasmic isoform of the protein Laforin (EPM2A) causes Lafora progressive myoclonus epilepsy. Hum. Mut. 23: 170-176.

Iwaki, T., Y. Hamada \& J. Tateishi. 1996. Advanced glycosylation end products and heat shock proteins acumulate in the basophilic degeneration of the myocardium and the corpora amylacea of the glia. Pathol. Int. 46: 757-763.

Ki, C.S., S.Y. Kong, D.W. Seo, S.B. Hong, H.J. Kim \& J.W. Kim. 2003. Two novel mutations in the EPM2A gene in a Korean patient with Lafora's progressive myoclonus epilepsy. J. Hum. Genet. 48: 51-54.

Kobayashi, K., K. Iyoda, Y. Ohtsuka, S. Ohtahara \& M. Yamada. 1990. Longitudinal clinocoelectrophysiologic study of a case of Lafora disease proven by skin biopsy. Epilepsia 31: 194-201.

Kufs, H. 1925. Uber eine spatform der amaurotischen idiotie und ihre heredofamiliaren grundlagen. Z. Ges. Neurol. Psychiat. 95: 169-188.

Lafora, G.R. 1911. Uber das vorkommen amyloider korperchen im innern der ganglienzellen; zugleich ein zum studium der amyloiden substanz im nervensystem. Virchows Arch [Pathol Anat] 205: 295-303

Lafora, G.R. \& B. Gluck. 1911. Beitrag zur histopathologie der myoklonischen epilepsie. Z. Ges. Neurol. Psychiat. 6: 1-14.

Liu, J. \& D.L. Brautigan. 2000. Insulin-stimulated phosphorylation of the protein phosphatase-1 striated muscle glycogen-targeting subunit and activation of glycogen synthase. J. Biol. Chem. 275: 1594015947.

Lundborg, H. 1903. Die progressive myoklonus epilepsy (Unverricht's Myoklonie). Almquist \& Wiksell, Upsala. pp. 1-207.

Man, Y.H., J.W Lin., W.H. Ju, G. Ahmadian, L. Liu, L.E. Becker, M. Sheng. \& Y.T. Wang. 2000. Regulation of AMPA receptor-mediated synaptic transmission by clathrin-dependent receptor internalization. Neuron. 25: 649-662.

Martell, K.J., T. Angelotti \& A. Ullrich. 1998. The "VH1like" dual-specificity protein tyrosine phosphatases. Mol. Cell 8: 2-11.

Minassian, B.A., J. Sainz \& A.V. Delgado-Escueta. 1996. Genetics of myoclonic and myoclonus epilepsies.1995-96. Clin. Neurosci. 3: 223-235.

Minassian, B.A., J.R. Lee, J.A. Herbrick, J. Huizenga, S. Soder, A.J. Mungall, I. Dunham, R. Gardner, C.Y. Fong, S. Carpenter, L. Jardim, P. Satishchandra, E. Andermann, O.C. Snead, I. Lopez-Cendes, L.C. Tsui, A.V. Delgado-Escueta, G.A. Rouleau \& S.W. Scherer. 1998. Mutations in a gene encoding a novel protein tyrosine phosphatase cause progressive myoclonus epilepsy. Nat. Genet. 20: 171-174.

Minassian, B.A., J. Sainz, J.M. Serratosa, M. Gee, L.G. Sakamoto, S. Bohlega, G. Geoffroy, C. Barr, S.W. Scherer, U. Tomiyasu, S. Carpenter, K. Wigg, A.V. Sanghvi \& A.V. Delgado-Escueta. 1999. Genetic locus heterogeneity in Lafora's progressive myoclonus epilepsy. Ann. Neurol. 45: 262-265.

Minassian, B.A., L. Ianzano, A.V. Delgado-Escueta \& S.W. Scherer. 2000a. Identification of new and commom mutations in the EPM2A gene in Lafora's disease. Neurology 54: 488-490.

Minassian, B.A., L. Ianzano, M. Meloche, E. Andermann, G.A. Rouleau, A.V. Delgado-Escueta \& S.W. Scherer. 2000b. Mutation spectrum and predicted function of Laforin in Lafora's progressive myoclonus epilepsy. Neurology 55: 341-346.

Minassian, B.A. 2001. Lafora's disease: Towards a clinical, pathologic, and molecular synthesis. Pediatr. Neurol. 25: 21-29.

Minassian, B.A., D.M. Andrade, L. Ianzano, E.J Young, E. Chan, C. A. Ackerkey \& S.W. Scherer. 2001. Laforin is a cell membrane and endoplasmic reticulum associated protein tyrosine phosphatase. Ann. Neurol. 49: 271-275.

Minassian, B.A. 2002. Progressive myoclonus epilepsy with polyglucosan bodies: Lafora Disease. Adv. Neurol. 89: 199-210. 
Neville, H.E., M.H. Brooke \& J.H. Austin.1974. Studies in myoclonus epilepsy (Lafora body form). Arch. Neurol. 30: $466-474$.

Nikaido, T., J. Austin \& H. Stukenbrok. 1971. Studies in myoclonus epilepsy. III: the effects of amylolytic enzymes on the ultrastructure of Lafora bodies. J. Histochem. Cytochem. 19: 382-385.

Nishimura, R.N., K.G. Ishak, R. Reddick, R. Porter, S. James \& J.A. Barranger. 1980. Lafora disease: diagnosis by liver biopsy. Ann. Neurol. $8: 409-415$.

Powell, H.C., H.W. Ward, R.S. Garrett, M.J. Orloff \& P.W. Lampert, 1979. Glycogen accumulation in the nerves and kidney of chronically diabetic rats. A quantitative electron microscopic study. J. Neuropathol. Exp. Neurol. 38: 114-127.

Raben, N., M. Danon, N. Lu, E. Lee, L. Shliselfeld, A.V. Skurat, P.J. Roach, J.C. Lawrence, O. Musumeci, S. Shanske, S. DiMauro \& P. Plotz. 2001. Surprises of genetic engineering: a possible model of polyglucosan body (Lafora) disease. Neurology 56: 1739-45.

Ramón y Cajal, S., A. Blanes, A. Martínez, E. Saenz \& M. Gutiérrez. 1975. Lafora's disease: an ultrastructural and histochemical study. Acta Neuropathol (Berl.) 30: 189-196.

Roach, P.J., Y. Cao, A. Corbett, A.A. DePaoli-Roach, I. Farkas, C.J. Fiol, H. Flotow, P.R. Graves, T.A. Hardy, T.W. Hrubey, E. Viskupic \& W. Zang. 1991. Glycogen metabolism and signal transduction in mammals and yeast. Adv. Enzyme Regul. 31: 101-120.

Robitaille, Y., S. Carpenter, G. Karpati \& S. DiMauro. 1980. A distinct form of adult polyglucosan body disease with massive involvement of central and peripheral neuronal processes and astrocytes: a report of four cases and a review of the occurrence of polyglucosan bodies in other conditions such a Lafora's disease and normal ageing. Brain 103: 315-336.

Roger, J., H. Gastaut, J. Boudouresques, M. Toga, D. Dubois \& H. Lob. 1967. Epilepsie-myoclonic progressive avec corps de Lafora. Etude clinique et polygraphique. Contrôle anatomique ultrastructural. Rev. Neurol. (Paris) $116:$ 197-212.

Roger, J., J.F. Pellissier, M. Bureau, C. Dravet, M. Revol \& P. Tinuper. 1983. Le diagnostic precoce de la maladie de Lafora. Importance des manifestations paroxystiques visuelles et interet de la biopsie cutanee. Rev. Neurol. (Paris) 139: 115-124.

Roger, J., P. Genton, M. Bureau \& C. Dravet. 1992. Progressive myoclonus epilepsies in childhood and adolescence. pp.381-400. In J. Roger, M. Bureau \& C. Dravet (eds.). Epileptic syndromes in infancy, childhood and adolescence. Libbey. London.

Sainz, J., B.A. Minassian, J.M. Serratosa, M.N. Gee, L.M. Sakamoto, R. Iranmanesh, S. Bohlega, R.J. Baumann, S. Ryan, R.S. Sparkes \& A.V. Delgado-Escueta. 1997. Lafora progressive myoclonus epilepsy: narrowing the chromosome $6 \mathrm{q} 24$ locus by recombinations and homozygosities. Am. J. Hum. Genet. 61: 1205-1209.

Sakai, M., J. Austin, F. Witmer \& L. Trueb. 1970. Studies in myoclonus epilepsy (Lafora body form) ; II. Polyglucosans in the systemic deposits of myoclonus epilepsy and in corpora amylacea. Neurology 20: 160-176.

Schmidt, A.M., S.D. Yan, S.F. Yan \& D.M. Stern. 2000. The biology of the receptor for advanced glycation end products and its ligands. Biochim. Biophys. Acta 1498: 99-111.

Serratosa, J.M., A.V. Delgado-Escueta, I. Posada, S. Shih, I. Drury, J. Berciano, J.A. Zabala, M.C. Antunez \& R.S. Sparkes. 1995. The gene for progressive myoclonus epilepsy of the Lafora type maps to chromosome 6q. Hum. Mol. Genet. 4: 1657-1663.

Serratosa, J.M., P. Gómez-Garre, M.E. Gallardo, B. Anta, D.B. de Bernabe, D. Lindhout, P.B. Augustijn, C.A. Tassinari, R.M. Malafosse, M. Topcu, D. Grid, C. Dravet, S.J. Berkovic \& S.R. de Cordoba. 1999. A novel protein tyrosine phosphatase gene is mutated in progressive epilepsy of the Lafora type (EPM2A). Hum. Mol. Genet. 8: 345-352.

Slack, F.J. \& G. Ruvkun. 1998. A novel repeat domain that is often associated with RING finger and B-box motifs. Trends Biochem. Sci. 23: 474-475.

Solís, V. 2000. Enfermedad de Lafora y efecto fundador en una pequeña localidad neotropical. Rev. Biol. Trop. 48: 703-706.

Steward, O. \& G.A. Banker. 1992. Getting the message from the gene to the synapse: sorting and intracellular transport of RNA in neurons. Trends Neurosci. 15: $180-186$. 
Toga, M., D. Dubois \& J. Hassoun. 1968. Ultra-structure des corps de Lafora. Acta Neuropathol. 10: 132-142.

Tonks, N.K. \& B.G. Nell. 1996. From form to function: signaling by protein tyrosine phosphatases. Cell 87: 365-368.

Unvericht, H. 1891. Die myoclonie. pp.1-128. Franz Deuticke, Viena.

Van Heycop Ten Ham, M.W. 1974. Lafora disease, a form of progressive myoclonus epilepsy. pp 382-422. In P.J. Vinken \& G.W. Bruyn (eds.). The Epilepsies. Handbook of Clinical Neurology Vol 15. North Holland Publishing Company. Amsterdam.

Villar-Palasi. C. \& J. J. Guinovart. 1997. The role of glucosa-6-phosphate in the control of glycogen synthase. FASEB J. 11: 544-558.

Wang, J, J.A Stuckey, M. J. Wishart \& J.E. Dixon. 2002. A unique carbohydrate binding domain targets the Lafora disease phosphatase to glycogen. J. Biol. Chem. 277: 2377-2380.
Woodgett, J.R. 1994. Regulation and functions of the glycogen synthase kinase-3 subfamily. Semin. Cancer Biol. 5: 269-275.

Yokoi, S. \& J. Austin. 1968. Studies in myoclonus epilepsy (Lafora body form). Arch. Neurol. 19: 15-33.

Yokoi, S., F. Austin, F. Witmer \& M. Sakai. 1968. Studies in myoclonus epilepsy (Lafora body form). 1. Isolation and preliminary characterization of Lafora bodies in two cases. Arch. Neurol. 19: 15-33.

Yokoi, S., H. Nakayama \& T. Negishi. 1975. Biochemical studies on tissues from a patient with Lafora disease. Clin. Chim. Acta 62: 415-423.

Zhao, W., H. Chen, H. Xu, E. Moore, N. Meiri, M.J Quon. \& D.L. Alkon. 1999. Brain insulin receptors and spatial memory: correlated changes in gene expression, tyrosine phosphorilation, and signaling molecules in the hippocampus of water maze trained rats. J. Biol. Chem. 274: 34893-34902. 